\title{
Lift Factor Analysis of Multifilamentary Coated Conductor Produced Using Two Level Undercut-Profile Substrates
}

Insinga, A. R.; Solovyov, M.; Usoskin, Alexander; Rutt, A.; Betz, U.; Lundeman, J. H.; Abrahamsen, A. B.; Grivel, J.-C.; Gömöry, F.; Wulff, A. C.

Published in:

IEEE Transactions on Applied Superconductivity

Link to article, DOI:

10.1109/TASC.2019.2896463

Publication date:

2019

Document Version

Peer reviewed version

Link back to DTU Orbit

Citation (APA):

Insinga, A. R., Solovyov, M., Usoskin, A., Rutt, A., Betz, U., Lundeman, J. H., Abrahamsen, A. B., Grivel, J-C., Gömöry, F., \& Wulff, A. C. (2019). Lift Factor Analysis of Multifilamentary Coated Conductor Produced U'sing Two Level Undercut-Profile Substrates. IEEE Transactions on Applied Superconductivity, 29(5), [8200704]. https://doi.org/10.1109/TASC.2019.2896463

\section{General rights}

Copyright and moral rights for the publications made accessible in the public portal are retained by the authors and/or other copyright owners and it is a condition of accessing publications that users recognise and abide by the legal requirements associated with these rights.

- Users may download and print one copy of any publication from the public portal for the purpose of private study or research.

- You may not further distribute the material or use it for any profit-making activity or commercial gain

- You may freely distribute the URL identifying the publication in the public portal 


\title{
Lift-Factor Analysis of Multifilamentary Coated Conductor Produced Using Two Level Undercut-Profile Substrates
}

\author{
A. R. Insinga, M. Solovyov, A. Usoskin, A. Rutt, U. Betz, J. H. Lundeman, \\ A. B. Abrahamsen, J.-C. Grivel, F. Gömöry and A. C. Wulff
}

\begin{abstract}
Here we present a detailed characterization of the critical current density of $\mathrm{YBa}_{2} \mathrm{Cu}_{3} \mathrm{O}_{7-x}$ (YBCO) two level undercut-profile substrate (2LUPS)-based samples made at the Technical University of Denmark (DTU) using Hastelloy substrate. The CC architecture was produced at Bruker HTS by means of alternating beam assisted deposition (ABAD) and pulsed laser deposition (PLD). The magnetization of different samples having all the same size, but with various numbers of filaments, including a non-filamentary reference sample have been measured in applied magnetic fields up to $16 \mathbf{T}$ and at temperatures ranging from $T=2 \mathbf{K}$ to $77 \mathbf{K}$.

A simple multiple rooftop model is proposed to estimate the critical current density of the filaments and it has been observed that filaments of $0.25 \mathrm{~mm}$ and $0.5 \mathrm{~mm}$ width are providing similar critical current densities in the order of $J_{c} \approx 2.3 \mathrm{MA} / \mathrm{cm}^{2}$ at $T=4.2 \mathbf{K}$ and $B=10 \mathbf{T}$ as well as the reference samples without filaments.

This result indicates that the filaments have been fabricated with no degradation of the superconductor and that the filaments are decoupled with non-superconducting material. Finally, the lift factor of the critical current $I_{c}$ with respect to $T=77 \mathrm{~K}$ and self field of the 2LUPS samples is estimated. This is reaching 10 at $T=4.2 \mathrm{~K}$ and $B=10$ Tesla.
\end{abstract}

\section{INTRODUCTION}

High temperature superconductors (SC) are being considered for applications where a good performance in highfield is required[1]. However, the hysteresis losses associated with AC applications need to be reduced by subdividing the SC tape into smaller filaments, and possibly transposition of the filaments as in Roebel cables or CORC cables[2]. The two level undercut-profile substrate (2LUPS) geometry is a way to subdivide the tape into filaments without reducing considerably the engineering-critical current[3], [4]. In this work we present a magnetic characterization approach based on vibrating sample magnetometry, and we apply the method to 2LUPS samples and non-filamented reference samples.

A. R. Insinga, J.-C. Grivel, and A. C. Wulff are with the Department of Energy Conversion and Storage, Technical University of Denmark, 4000 Roskilde, Denmark.

M. Solovyov, and F. Gömöry are with the Institute of Electrical Engineering SAS, Slovak Academy of Sciences, 84104 Bratislava, Slovak Republic

A. Usoskin, A. Rutt, and U. Betz are with Bruker HTS GmbH, 63755 Alzenau, Germany.

J. H. Lundeman is with SUBRA IVS, 2765 Smørum, Denmark.

A. B. Abrahamsen is with the Department of Wind Energy, Technical University of Denmark, 4000 Roskilde, Denmark.

Color versions of one or more of the figures in this paper are available online at http://ieeexplore.ieee.org.
Many of the measurement techniques commonly employed for characterization of SC tapes present additional challenges when applied to filamented tapes. As we discuss here, VSM measurement is a viable possibility for rapidly evaluating the performance of a small sample in different conditions including very intense fields, and low temperatures, i.e. close to the real operating conditions of many relevant magnet applications. Some care is necessary when interpreting VSM data of filamented samples and when comparing samples with different filament widths. Assuming some realistic approximations, it is possible to estimate the critical current density field-dependence from the magnetic moment signal obtained by VSM measurement of filamented samples.

\section{Methods}

\section{A. Fabrication}

The 2LUPS geometry, shown in Fig. 1(a), consists of two levels of vertically separated plateaus which are realized by means of electrochemical etching.

When the buffer layers and the YBCO layer are deposited onto the substrate, the 2LUPS geometry causes the SC layers of the upper plateaus to be decoupled from the SC layers of the adjacent lower plateaus, as illustrated in Fig. 1(b). The resulting tape is composed by many narrow filaments directed along the tape rolling direction $x$. The subdivision into filaments is especially advantageous for AC applications, as it reduces the hysteresis losses [14], and for strong DC magnets, as it increases field stability[15]. In this study we compare two different 2LUPS tapes, characterized by filaments-widths of $0.25 \mathrm{~mm}$ and $0.5 \mathrm{~mm}$, as well as two non-filamentary reference tapes. The $\mathrm{CC}$ architecture was realized at Bruker HTS employing alternating beam assisted deposition (ABAD) and pulsed laser deposition (PLD), finalized by Ag capping layer[5]. Complete coverage with $\mathrm{Ag} / \mathrm{Cu}$ is beneficial for $\mathrm{DC}$ magnet stabilization.

\section{B. VSM Measurement}

An approximately square sample of $5 \mathrm{~mm}$ side length has been cut from each tape for VSM analysis. The characterization has been performed using an vibrating sample magnetometer in a cryogenic-free measurement system. For each sample the characterization has been performed at different temperatures $T$ spanning from $2 \mathrm{~K}$ to $77 \mathrm{~K}$, and with field magnitude up to $16 \mathrm{~T}$. The applied field is normal to the 


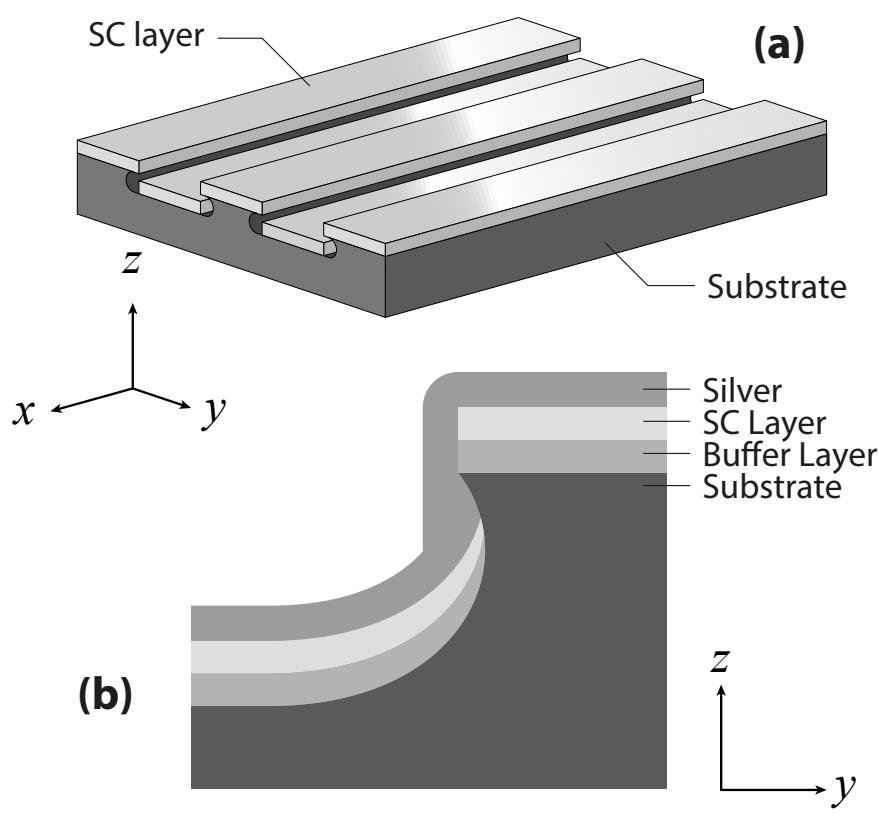

Fig. 1. 2LUPS geometry. (a): portion of a tape having three upper plateaus separated by two lower plateaus. (b): cross section showing the decoupling between SC layer of the upper and lower plateaus achieved by the undercut profile.

surface of the SC layer, i.e. running along the $z$ direction. The $z$ component of the total magnetic moment of the sample is thus measured as the applied field is varied and the temperature is kept constant.

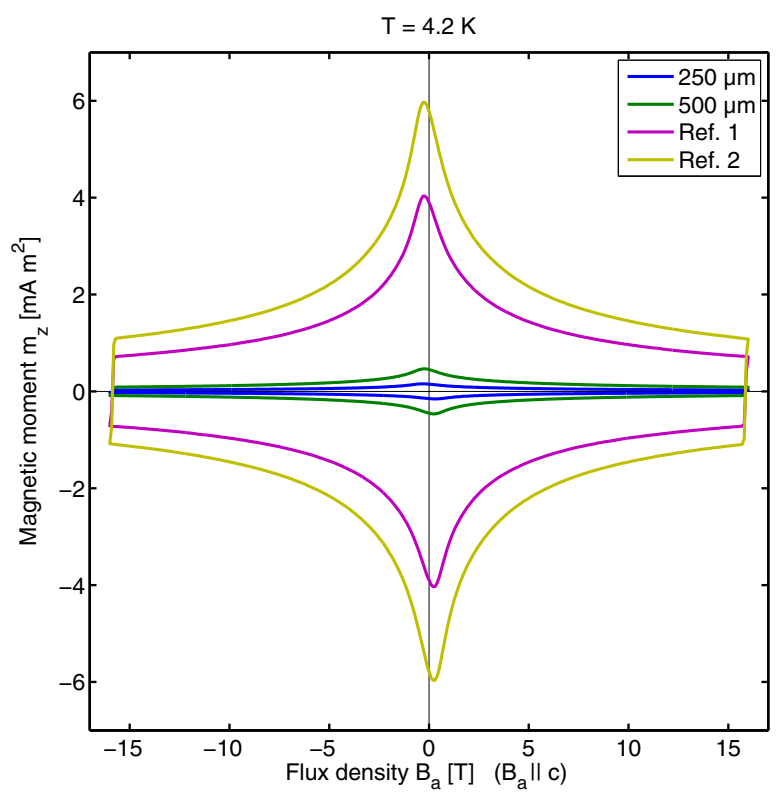

Fig. 2. Comparison between the hysteresis loops of the different samples at $T=4.2 \mathrm{~K}$. The two reference samples almost give the same response (the difference is due to the slightly different dimensions of the samples). Because of the subdivision into filaments the 2LUPS samples generate a much lower signal.
As example, the curves corresponding to $T=4.2 \mathrm{~K}$ are shown in Fig. 2. Since the dimensions of the two reference samples are not exactly identical, they generate a magnetic moment with slightly different magnitude. The magnetic moment generated by the 2LUPS samples is considerably less intense; the $0.25 \mathrm{~mm}$ sample is the one producing the smallest signal. This behavior is expected, and can be explained by the subdivision into electrically decoupled filaments.

\section{Removal of base signal}

Since we wish to isolate the magnetic moment generated by the screening currents running in the SC layer, we need to remove the baseline signal corresponding to the ferromagnetic or paramagnetic response. The baseline signal is primarily due to the ferromagnetic response of the substrate.

In order to subtract the baseline signal, we remove from each data-point the middle point between the upper and lower curves of the hysteresis loop. This operation is equivalent to considering the vertical distance $\Delta m=\left(m_{\uparrow}-m_{\downarrow}\right)$ between the upper and lower branches. As explained in Sec. II-D, this procedure is justified when the high-field approximation is assumed, since in this limit the curve is expected to be perfectly symmetrical with respect to a vertical reflection.

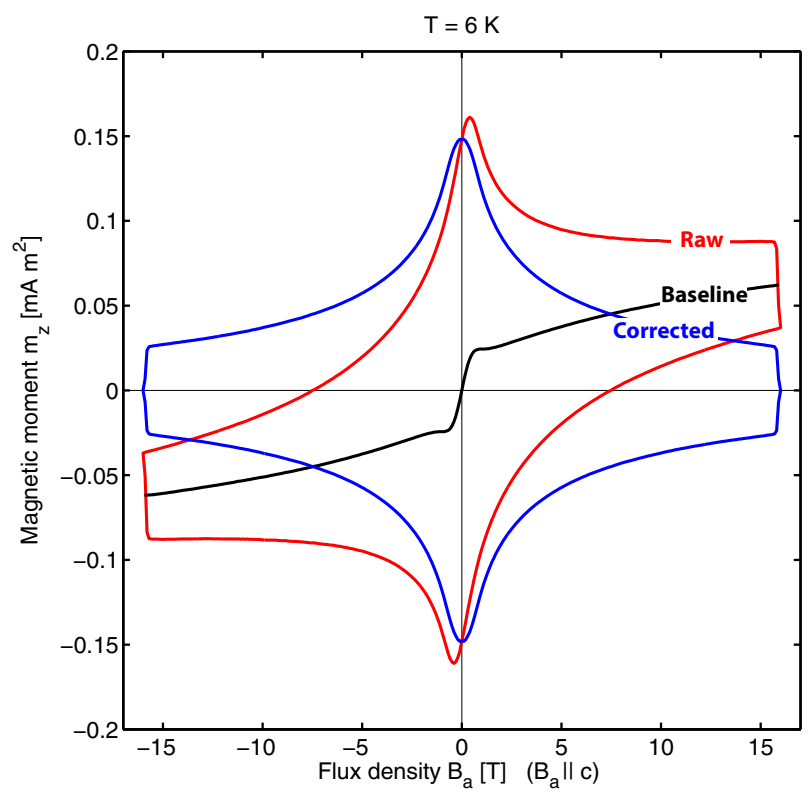

Fig. 3. Removal of substrate magnetic response. The red curve is the original hysteresis loop. The black curve is the baseline signal, i.e. the middle point between the upper and lower branches of the loop. The blue curve is the difference which is assumed to be the magnetic moment generated by the SC currents.

An example of corrected hysteresis loop is shown in Fig. 3. The raw data corresponds to the red curve, the baseline curve to the black curve and the corrected loop to the blue curve. This example corresponds to the $0.25 \mathrm{~mm}$ sample at $6 \mathrm{~K}$. 


\section{Data interpretation}

The magnetic behavior of superconducting material is modeled by assuming a microscopic $\boldsymbol{J}-\boldsymbol{E}$ relation between the electric field $\boldsymbol{E}$ and the current density $\boldsymbol{J}$. Commonly it is assumed that the vectors $\boldsymbol{J}$ and $\boldsymbol{E}$ are aligned, and that the relation between their magnitudes obeys the following power law[11], [12]:

$$
\left(J / J_{c}\right)^{n}=\left(E / E_{c}\right)
$$

The critical field $E_{c}$ is set to $10^{-6} \mathrm{~V} / \mathrm{cm}$, and the critical current $J_{c}$ depends on the magnetic flux density $\boldsymbol{B}$ and the temperature. In general, the resulting set of partial differential equations can only be solved by numerical approaches.

However, in the conditions of a high-field VSM measurement, it is realistic to simplify the treatment by considering approximate models.

- The critical state model (Bean's model)[6], [7], corresponding to the limit $n \rightarrow \infty$, where $n$ is the exponent appearing in the power law expressing the $\boldsymbol{E}$ - $\boldsymbol{J}$ relation. In this limit the process is still history-dependent, but rate-independent.

- High field limit - the magnitude $B_{a}$ of the applied magnetic flux density is much more intense than the magnitude of the flux density $\boldsymbol{B}_{J}$ generated by the screening currents. The norm of the current is thus determined directly by $B_{a}$ via the $J_{c}(B)$ relation. The magnetic flux density is thus assumed to be always normal to the superconducting layer.

- Electrical decoupling - with respect to the super-currents, the filaments are considered perfectly electrically insulated from each other: the continuity equation applies separately to each filament. Eddy currents in the Ag layer are not considered.

- Rooftop model - we assume that the current distribution is given by the rooftop model[8], [9], [10]: the current is always parallel to one of the sides of the rectangular filaments as shown in Fig. 4.

Applying these approximations we can readily interpret the VSM data. Denoting by $\boldsymbol{x}$ a generic point of space and by $\boldsymbol{J}(\boldsymbol{x})$ the current density in that point, the magnetic moment $\boldsymbol{m}$ about the origin is defined as

$$
\boldsymbol{m}=(1 / 2) \iiint_{\Omega} \boldsymbol{x} \times \boldsymbol{J}(\boldsymbol{x}) d V
$$

where the integration domain $\Omega$ extends over the region occupied by the sample. Assuming the current distribution corresponding to the rooftop model, the $z$ component of the total magnetic moment is [8]

$$
m_{z}=(1 / 2) d J_{c} \sum_{i}\left(\left(b a_{i}^{2}\right) / 2-a_{i}^{3} / 6\right),
$$

where we denoted by $a_{i}$ the width of each filament, by $b$ their length, and by $d$ their thickness.

We measured from high-magnification pictures the individual widths $a_{i}$ of each filament and the length $b$ of each of the VSM samples. As shown in Fig. 4, we estimated a spacing

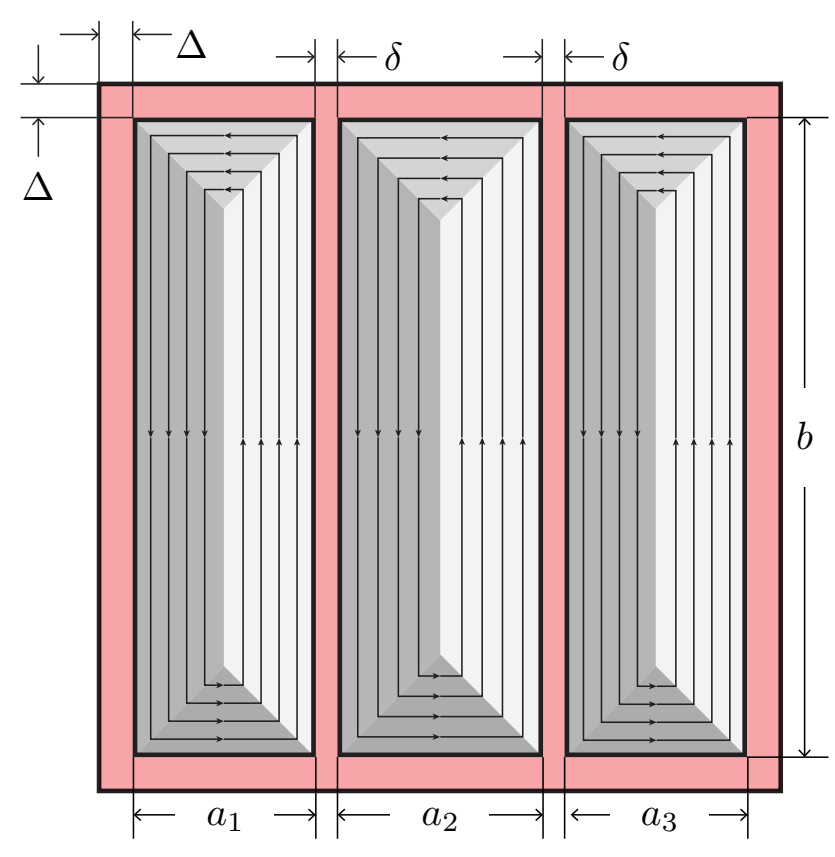

Fig. 4. The roof-top model has been employed to calculate the proportionality factor between magnetic moment and critical current. The current distribution assumed by this model is shown in the figure. The spacing $\delta$ between the filaments and the external border $\Delta$ are assumed to be non-functional.

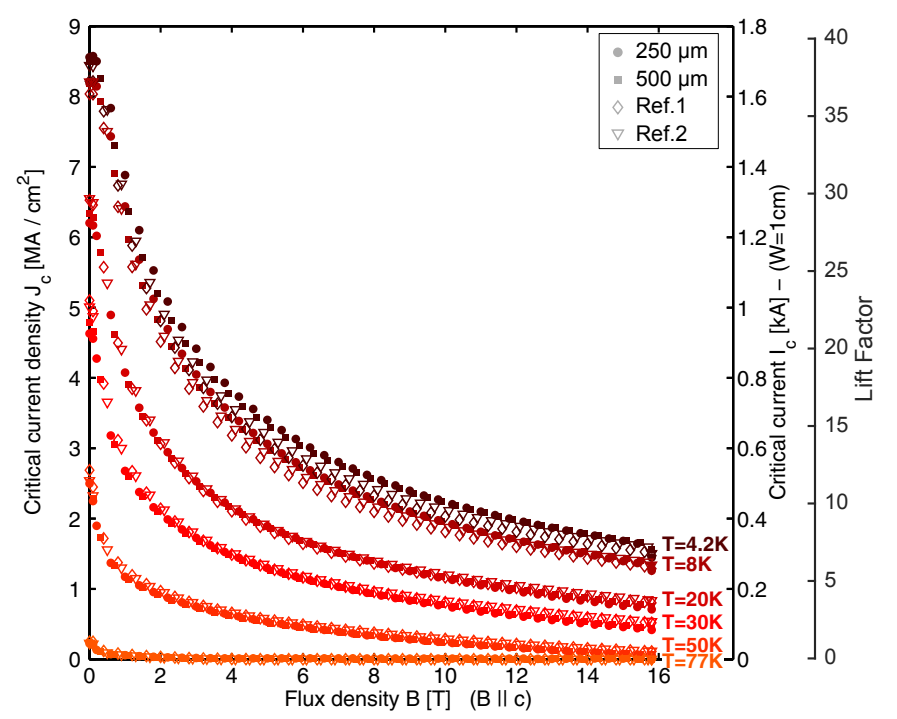

Fig. 5. Critical current density $J_{c}(B)$ curves of filaments in 2LUPS sample calculated from the VSM data applying Eq. 3. A superconductor layer thickness of $d=2 \times 10^{-6} \mathrm{~m}$ is assumed for the $J_{c}(B)$ estimate (left) and a tape width of $1 \mathrm{~cm}$ is assumed for the critical current $I_{c}(B)$ (right). Two reference samples (Ref.1 and Ref.2) without filamentation are included for comparison.

of $\delta=0.06 \mathrm{~mm}$ between the filaments, and a damaged area of thickness $\Delta=0.1 \mathrm{~mm}$ running along the external border of the sample. We assumed that the thickness of the superconducting layer is given by $d=2 \times 10^{-6} \mathrm{~mm}$ [5]. These values are used to compute $J_{c}$ from $m$ employing Eq. 3 . 
The critical current $I_{c}$ corresponding to a $1 \mathrm{~cm}$ wide tape is obtained by multiplying $J_{c}$ by $d$ and $W=1 \mathrm{~cm}$.

The resulting curves are shown in Fig. 5. The colors indicate the temperature and the different shapes of the markers corresponds to different samples. For each temperature all the $J_{c}$ curves corresponding to the different samples are welloverlapped to each other.

The lift factor of a tape at temperature $T$ and with applied field $B$ is the total current transported by the tape in such conditions, normalized by the current $I_{0}$ carried by the same tape at $77 \mathrm{~K}$ in self-field. In order to calculate the lift factor we estimate $I_{0}$ from the VSM data, obtaining the value $I_{0}=54 \mathrm{~A}$ for a $1 \mathrm{~cm}$ wide tape. Applying this normalization we calculated the lift factors shown on the right-hand side of Fig. 5.

\section{Discussion AND CONCLUSION}

Similarly to the hysteresis losses in AC applications, the magnetic response of a tape to the time-varying field applied during a VSM measurement decreases when the number of filaments increases [13], [14]. On the contrary, the net current transported by a tape in DC applications is expected to be almost the same as for a non-filamented sample.

In this paper we discussed a simple way to estimate the in-field critical current of filamented tapes from VSM data [8]. As can be seen from Fig. 5, the critical current density of the filaments of the 2LUPS samples is independent of the filament width, and is also similar to the non-filamented reference samples.

This observation is indicating that the filamentation is not causing a severe decrease of the superconducting properties except from the edge region. Secondly, the results seem to indicate that the underlying approximations are correct, and that all the filaments are decoupled by non-superconducting materials.

Other possible collateral causes for the lower VSM signal given by filamented tapes could be the presence of defects in the surface of the Hastelloy substrate [3], or damaging of the SC layer occurred during cutting of the VSM samples. One possible approach that would further corroborate the procedure would be to perform transport measurement in DC conditions and compare the result with the VSM signal. Such an investigation would confirm that the reduction of the VSM signal is entirely due to the subdivision into filaments.

\section{ACKNOWLEDGMENT}

A.R.I. and A.C.W. acknowledge the financing by the ATOMIS DFF-Research Project which is funded by the Danish Council for Independent Research (project ID DFF-611100252).

\section{REFERENCES}

[1] C. Senatore, M. Alessandrini, A. Lucarelli, R. Tediosi, D. Uglietti and Y. Iwasa, Progresses and challenges in the development of high-field solenoidal magnets based on RE123 coated conductors, Supercond. Sci. Technol., vol. 27, no. 10, Sept. 2014, Art. ID. 103001 1-26.
[2] W. Goldacker, F. Grilli, E. Pardo, A. Kario, S. I Schlachter and M. Vojenčiak, Roebel cables from REBCO coated conductors: a one-centuryold concept for the superconductivity of the future, Supercond. Sci. Technol., vol. 27, no. 9, Aug. 2014, Art. ID. 093001.

[3] A. C. Wulff, M. Solovyov, F. Gömöry, A. B. Abrahamsen, O. V. Mishin, A. Usoskin, A. Rutt, J. H. Lundeman, K. Thydén, J. B. Hansen and J.-C. Grivel, Two level undercut-profile substrate for filamentary $\mathrm{YBa} 2 \mathrm{Cu} 3 \mathrm{O} 7$ coated conductors, Supercond. Sci. Technol., vol. 28, May 2015, Art. ID. 072001.

[4] A. C. Wulff, J. H. Lundeman, J. B. Hansen, O. V. Mishin, Y. Zhao, R. Mohajeri and J.-C. Grivel, A two level undercut-profile substrate for chemical-solution-based filamentary coated conductors, IEEE Trans. on Appl. Supercond., vol. 26, no. 3, Apr. 2016, Art. ID. 6601604 1-4.

[5] A. Usoskin, U. Betz, F. Hofacker, A. Rutt, K. Schlenga, B. Prause, L. Rossi, L. Bottura, A. Ballarino, C.Senatore, A. Kario, W. Goldacker, A. Meledin, D. Abraimov, D. Larbalestier, Double-Disordered HTS Coated Conductors and Their Assemblies Aimed for Ultra-High Fields: Large Area Tapes, IEEE Trans. on Appl. Supercond., vol. 28, no. 4, Feb. 2018, Art. ID. 6602506 1-6.

[6] C. P. Bean, Magnetization of Hard Superconductors, Phys. Rev. Lett., vol. 8, no. 6, Mar. 1962, 250-253.

[7] C. P. Bean, Magnetization of High-Field Superconductors, Rev. Mod. Phys., vol. 36, Mar. 1964, 31-39.

[8] E. H. Brandt, Electric field in superconductors with rectangular cross section, Phys. Rev. B, vol. 52, no. 21, Dec. 1999, 442-457.

[9] E. H. Brandt, Square and rectangular thin superconductors in a transverse magnetic field, Phys. Rev. Lett., vol. 74, no. 15, Apr. 1995, 3025-3028.

[10] L. Prigozhin, Solution of Thin Film Magnetization Problems in Type-II Superconductivity, J. Comput. Phys., vol. 144, no. 1, Jul. 1998, Art. ID. CP985978 180-193.

[11] V. M. R. Zermeno, A. B. Abrahamsen, N. Mijatovic, B. B. Jensen and M. P. Sørensen, Calculation of alternating current losses in stacks and coils made of second generation high temperature superconducting tapes for large scale application, J. Appl. Phys., vol. 114, no. 17, Nov. 2013, Art. ID. 173901 1-8.

[12] E. Pardo, J. Šouc and L. Frolek, Electromagnetic modelling of superconductors with a smooth current-voltage relation: variational principle and coils from a few turns to large magnets, Supercond. Sci. Technol., vol. 28, no. 4, Feb. 2015, Art. ID. 044003.

[13] M. Osipov, E. Splavnik, S. Pokrovskii, A. Andreev, N. Mineev, D. Abin and I. Rudnev, Investigation of the hysteresis losses in ĐaĐa tapes after laser filamentation, J. Phys.: Conf. Ser., vol. 747, no. 1, Sept. 2016, Art. ID. 012044 1-3.

[14] M. D. Sumption, E. Lee, Coleman B. Cobb, Paul N. Barnes, Timothy J. Haugan, Justin Tolliver, Charles E. Oberly and E. W. Collings Hysteretic Loss vs . Filament Width in Thin YBCO Films Near the Penetration Field, IEEE Trans. on Appl. Superc., vol. 13, no. 2, Jun. 2013, 3553 - 3556.

[15] Y. Yanagisawa, Y. Xu, X. Jin, H. Nakagome, and H. Maeda, Reduction of Screening Current-Induced Magnetic Field of REBCO Coils by the Use of Multi-Filamentary Tapes, IEEE Trans. on Appl. Superc., vol. 25, no. 3, Jun. 2015, Art. ID. 6603705 1-5. 